\title{
The slippery context effect in psychophysics: Intensive, extensive, and qualitative continua
}

\author{
LAWRENCE E. MARKS \\ John B. Pierce Laboratory and Yale University, New Haven, Connecticut
}

\begin{abstract}
When subjects gave magnitude estimates of 500 - and $2500-\mathrm{Hz}$ tones at various SPLs, they judged a $500-\mathrm{Hz}$ tone of $60 \mathrm{~dB}$ to be as loud as a $2500-\mathrm{Hz}$ tone of $57 \mathrm{~dB}$ in one context (low SPLs at $500 \mathrm{~Hz}$, high SPLs at $2500 \mathrm{~Hz}$ ), but as loud as a $2500-\mathrm{Hz}$ tone at $40 \mathrm{~dB}$ in another context (high SPLs at $500 \mathrm{~Hz}$, low at $2500 \mathrm{~Hz}$ ) (Marks, 1988). Such shifts in matches derived from judgments of multidimensionally varying stimuli are termed slippery context effects. The present set of seven experiments showed that slippery effects were absent from judgments of pitch of tones at different loudnesses, duration of tones at different pitches, and length of lines at different colors, though a small effect emerged in judgments of duration of tones and lights. Slippery context effects were substantial when subjects gave magnitude estimates of loudness of 500 - and $2500-\mathrm{Hz}$ tones under conditions in which the pitch at each trial either was cued visually beforehand or could be known through the regular stimulus sequence, and with instructions to make absolute magnitude estimates. The results are consistent with the view that slippery context effects occur automatically and "preattentively."
\end{abstract}

How do judgments of perceived intensity or quality depend on the set of possible stimuli in the ensemble, on what Garner (1974) has called the "inferred set'? Decades of research in the tradition of Helson (1964), for example, have shown that psychophysical judgments depend on the range and spacing of stimuli, and on the relative frequencies with which the stimuli are presented. Parducci (1965) has modeled responses in terms of a range-frequency model, which assumes that when subjects judge stimuli on rating scales, they tend to use the available response categories equally often. Judgments also reveal sequential dependencies: The response to the current stimulus depends on stimuli and responses on the previous trial or trials (see, e.g., Cross, 1973; Jesteadt, Luce, \& Green, 1977; Lockhead \& Hinson, 1986; Ward, 1979). Such context effects are virtually universal, appearing in a wide variety of procedures (bounded rating scales, magnitude estimation, absolute identification) and perceptual continua.

Over the past several years, my colleagues and I have been studying contextual effects within a double shiftingcontext paradigm. This paradigm varies not one dimension, as in conventional scaling experiments, but two: a criterial dimension, whose values the subject judges, and

This research was supported by NIH Grants DC00271 and DC00818 to Lawrence Marks and NIH Grant RR05692 to the Pierce Laboratory. I thank Mary Murray, Patricia O'Neill, Jennifer Scott, and David Wiesenfeld for their assistance in running the experiments and analyzing the data, and Daniel Algom, Robert Melara, Bonnie Potts, Martha Teghtsoonian, and an anonymous referee for their comments on an earlier version of the paper. Please address correspondence to Lawrence $E$. Marks, John B. Pierce Laboratory, 290 Congress Avenue, New Haven, CT 06519. a vehicle dimension, which serves as the carrier for the contextual variation. For example, subjects might judge the loudness (criterial dimension) of tones varying in sound frequency or pitch (vehicle), as well as soundpressure level (SPL). In the double shifting-context paradigm, two contextual conditions are presented. In one condition, the low-frequency tones take on low SPLs and the high-frequency tones take on high SPLs; in the other condition, the pairing of frequency with SPLs reverses.

This paradigm produces what Marks and Warner (1991) call a slippery context effect-a change in the relation between responses to the two sets of stimuli as a function of the change in pairing of frequency and SPL. Figure 1 shows such an effect (Marks, 1988). Subjects judged the loudness of 500 - and $2500-\mathrm{Hz}$ tones; in one condition, the $500-\mathrm{Hz}$ tones were low in SPL and the $2500-\mathrm{Hz}$ tones high, and in the other condition, the pairing was reversed. In the first condition (left side of Figure 1), equal SPLs at the two frequencies produced roughly equal responses; but in the second condition (right side), the $2500-\mathrm{Hz}$ tones were judged much greater in loudness. The total change across conditions in the intensity levels of the stimuli, $\Delta C$, was $40 \mathrm{~dB}$, and the consequent shift in matching SPLs was about $17 \mathrm{~dB}$, or nearly half of $\Delta C$.

Similarly large slippery context effects have been obtained when the criterial dimension was sensory intensity and the vehicle was quality varied through sensory modality (i.e., variations in intensity were carried by hearing and vision, by hearing and touch, or by hearing and taste; see Marks, 1991a; Marks, Szczesiul, \& Ohlott, 1986; Marks et al., 1988). On the other hand, effects were small when the criterial dimension was loudness and the vehicle was spatial location (left ear and right ear; Marks, 1988), and when the criterial dimension was duration and 


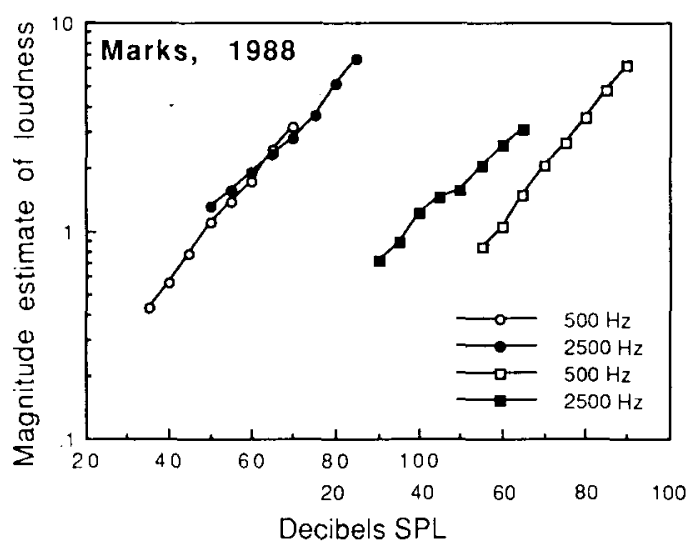

Figure 1. Geometric average magnitude estimates of the loudness of 500-Hz tones (open symbols) and 2500-Hz tones (filled symbols), plotted against sound-pressure level (SPL). In Condition A (circles, left), the SPLs at $500 \mathrm{~Hz}$ were low and those at $2500 \mathrm{~Hz}$ were high; in Condition B (squares, right), the SPLs at $500 \mathrm{~Hz}$ were high and those at $2500 \mathrm{~Hz}$ were low. (Data are from Marks, 1988, Experiment 3.)

the vehicle dimension was modal quality (flashes of light and pulses of tone).

Thus the context effects that emerge in the double shifting-context paradigm are called slippery both because they entail a slippage of two stimulus-response functions relative to each other and because the size of the effect depends on the joint choice of criterial dimension and vehicle.

The existence of slippery context effects serves to refute the notion that pairs of stimuli judged to be equal in perceptual magnitude largely maintain their equality across changes in stimulus context; thus slippery context effects limit or constrain perceptual invariances. Moreover, growing evidence suggests that these effects represent changes in underlying percepts themselves, not just changes in multiple response scales. For example, Schneider and Parker (1990) found slippery context effects when subjects were asked simply to indicate on each trial which of two pairs of $500-$ and $2500-\mathrm{Hz}$ tones differed more in loudness; changing contexts across sessions changed the matching SPLs. Using magnitude estimation, Marks and Warner (1991) found no slippery effects when all of the tones fell roughly within a single critical band. Finally, Marks (1991b) reported context-based changes in direct loudness matches between $500-$ and $2500-\mathrm{Hz}$ tones; there, the matching trials were embedded within the double shiftingcontext paradigm, in which subjects also gave magnitude estimates of the loudness of individual tones.

The present study addresses two main questions. First, to date, nearly all studies of slippery context effects have focused on judgments of sensory intensity; the present study asks whether analogous effects characterize judgments of sensory quality and spatial and temporal extent. Do similar effects result when criterial dimension and vehicle are reversed as, for example, when subjects judge pitch (criterial dimension) at different loudnesses (vehicle)? Second, it is not clear to what extent, if any, slippery context effects may be reduced or eliminated through focusing subjects' attention to the criterial dimension, thereby reducing uncertainty on each trial about the value on the vehicle dimension, or through using scaling procedures claimed to minimize response biases. If, for example, slippery context effects take place automaticallypreattentively-and represent changes in underlying percepts, robust effects should remain despite focusing of attention or changes in response scale.

\section{EXPERIMENT 1 Pitch at Two Loudnesses}

Substantial slippery context effects appear when subjects judge the perceived intensities of qualitatively different stimuli drawn either from different modalities or from the same modality. For example, implicit loudness matches shift when subjects judge the loudnesses of low- and highfrequency tones that vary in SPL (Marks, 1988; Schneider \& Parker, 1990). The first experiment turned the paradigm around, inverting criterial dimension and vehicle by asking subjects to judge the pitches of soft and loud tones that varied in sound frequency. In one condition, subjects judged the pitches of a stimulus ensemble containing relatively low-frequency tones that were soft and highfrequency tones that were loud; in the other condition, subjects judged the pitches of an ensemble in which lowfrequency tones were loud and high-frequency tones were soft.

\section{Method}

Stimull. Stimuli were pure tones, produced by Coulbourn Instruments modules under the control of an Apple Ile computer and delivered binaurally through calibrated TDH-39 headphones. Each tone lasted $1 \mathrm{sec}$, with rise and decay of $10 \mathrm{msec}$.

Two versions of Experiment 1 were conducted, the first with 10 and the second with 12 sound frequencies in all. In Experiment $1 \mathrm{~A}$, there were two overlapping sets of 8 : a low-frequency set of $250,375,500,625,750,880,1020$, and $1180 \mathrm{~Hz}$, and a high-frequency set of $500,625,750,880,1020,1180,1375$, and $1620 \mathrm{~Hz}$. Thus six frequencies were common to the two sets. Moreover, each set of frequencies could be low in intensity $(50 \mathrm{~dB})$ or high $(80 \mathrm{~dB})$. Condition $\mathrm{A}$ comprised the low-frequency stimuli at $50 \mathrm{~dB}$ and the high-frequency stimuli at $80 \mathrm{~dB}$; Condition $B$ reversed the pairing.

In Experiment 1B, there were 12 sound frequencies, also divided into two overlapping sets of 8: a low-frequency set containing 150, $190,225,285,345,430,515$, and $650 \mathrm{~Hz}$, and a high-frequency set containing 345, 430,515, 650,770, 990, 1500, and $1900 \mathrm{~Hz}$. Hence, in Experiment 1B, 4 frequencies were common to the low and high sets. Again, Condition A comprised the low-frequency stimuli at $50 \mathrm{~dB}$ plus the high-frequency stimuli at $80 \mathrm{~dB}$, whereas Condition $B$ comprised the low frequencies at $80 \mathrm{~dB}$ plus the high frequencies at $50 \mathrm{~dB}$.

The two versions of Experiment 1 differed also in the way stimuli were equated for low or high loudness. In Experiment 1A, the stimuli were set to 50 or $80 \mathrm{~dB}$ by means of the A-weighting scale. In Experiment 1B, the stimuli were equated to the loudness of $1000-\mathrm{Hz}$ tones at 50 and $80 \mathrm{~dB}$ by setting the levels in accord with a set of previously determined, average equal-loudness matches. 
Procedure. The method was magnitude estimation. Subjects were instructed to assign to the first stimulus whatever number they deemed appropriate to stand for the pitch; then, for succeeding tones, the subjects were to assign other numbers in proportion to pitch, using integers and decimal numbers as needed. The subjects fed their numbers through a keypad to the computer.

After the first stimulus, which was not included in the results, each of the 16 stimuli of the condition, A or B, was presented a total of 16 times, under the constraint that every stimulus follow every stimulus including itself exactly once (see Cross, 1973; Marks, 1988, Experiment 3), but otherwise in random order. Each subject served in two sessions, one of Condition $A$ and the other Condition B, held on different days. Half served first in A and then in B, half in B and then in A. Sixteen subjects served in Experiment $1 \mathrm{~A} ; 3$ of those subjects, plus 13 new ones, served in Experiment $1 \mathrm{~B}$.

The subjects were recruited largely from the general Yale community and were paid to participate. Because many of the subjects had moderate or considerable musical experience, I administered to each subject a questionnaire assessing the degree of the subject's musical background, in order to see whether degree of musical experience might correlate with psychophysical performance in judging pitch. It didn't.

\section{Results and Discussion}

Magnitude estimates given to each stimulus were averaged arithmetically across replicates and geometrically across subjects within each contextual condition; they are plotted in Figures 2 (Experiment 1A) and 3 (Experiment 1B). These results have two main features, which are much the same for both experiments. First, at each sound frequency, there is a small overall difference between pitch judgments of soft and loud tones; in both contextual conditions of each experiment, the function for loud tones lies slightly above the corresponding function for soft tones. On the average, the ratio of pitch judgments for loud and soft tones was 1.071 in Experiment 1A and 1.044 in Experiment 1B. Although the difference between loud and soft tones failed to reach statistical reliability in either experiment alone [in Ex-

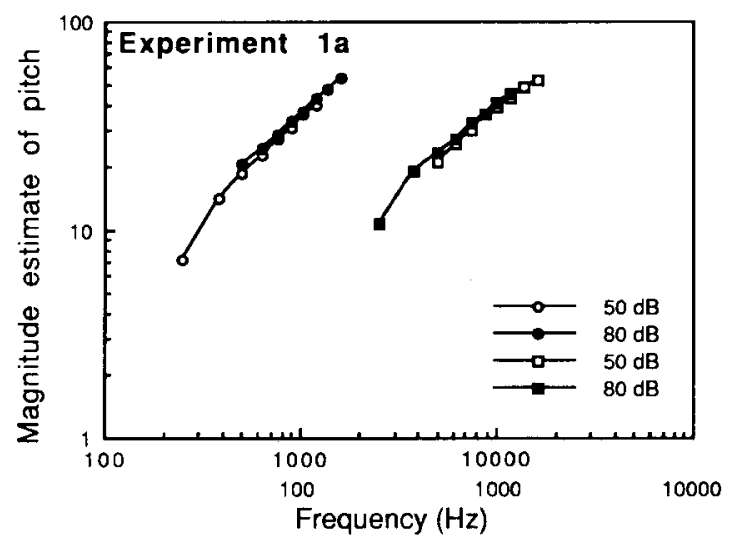

Figure 2. Experiment 1A: Geometric average magnitude estimates of the pitch of 50-dB tones (open symbols) and 80-dB tones (filled symbols), plotted against sound frequency. In Condition A (circles, left), the frequencies at $50 \mathrm{~dB}$ were low and those at $80 \mathrm{~dB}$ were high; in Condition $B$ (squares, right), the frequencies at $50 \mathrm{~dB}$ were high and those at $80 \mathrm{~dB}$ were low.

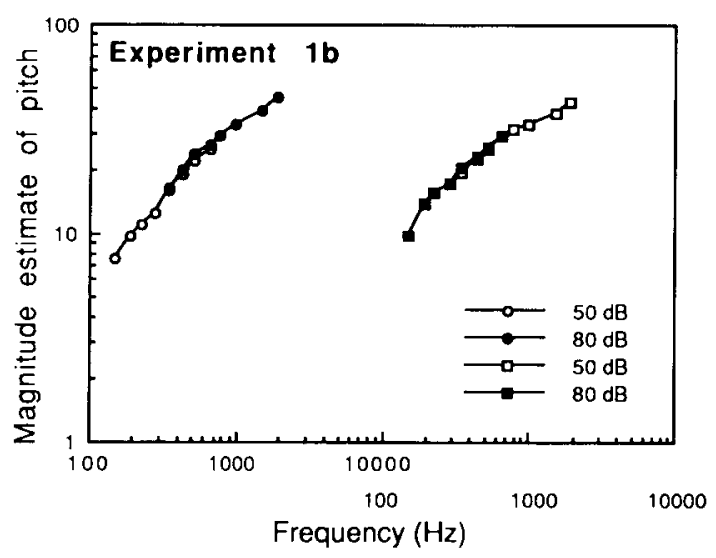

Figure 3. Experiment 1B: Geometric average magnitude estimates of the pitch of 50-dB tones (open symbols) and 80-dB tones (filled symbols), plotted agninst sound frequency. In Condition A (circles, left), the frequencies at $50 \mathrm{~dB}$ were $\mathrm{low}$ and those at $80 \mathrm{~dB}$ were high; in Condition B (squares, right), the frequencies at $50 \mathrm{~dB}$ were high and those at $80 \mathrm{~dB}$ were low.

periment $1 \mathrm{~A}, t(15)=1.77, p=.097$; in Experiment 1B, $t(15)=1.41, p=.178]$, the difference becomes reliable if the results of the two experiments are pooled (and a single average value is taken for each of the three subjects who served in both experiments) $[t(28)=2.45, p=$ $.023]$. Note that this small tendency for greater intensity to yield greater judgments of pitch differs from the psychophysical interaction between frequency and intensity in determining pitch (see, e.g., Stevens, 1935), where raising the intensity of low-frequency tones $(<1000 \mathrm{~Hz})$ decreases pitch but raising the intensity of high-frequency tones $(>1000 \mathrm{~Hz})$ increases pitch. In the present case, I suspect that subjects simply tended to "integrate" loudness with pitch; in Garner's (1974) terminology, subjects treated pitch and loudness to some extent as integral dimensions (cf. Grau \& Kemler Nelson, 1988; Melara \& Marks, 1990).

The second, and most important, feature of the results is that judgments of pitch showed no slippery context effect. Except for the slight multiplication of pitch by loudness, which was essentially the same in both contextual conditions, sound frequency determined pitch regardless of the loudness context. This outcome stands in marked contrast with the substantial slippery context effect evident when subjects judged loudness at different sound frequencies (compare Figures 2 and 3 to Figure 1). Reversing the variables of criterial dimension and vehicle yielded a markedly different result. At least under the present circumstances, pitch seems impervious to slippery context effects. ${ }^{1}$

\section{EXPERIMENT 2 Duration at Two Pitches}

In Experiment 2, I sought to learn whether magnitude estimates show a slippery context effect when subjects judge the duration of low- and high-frequency tones. For 


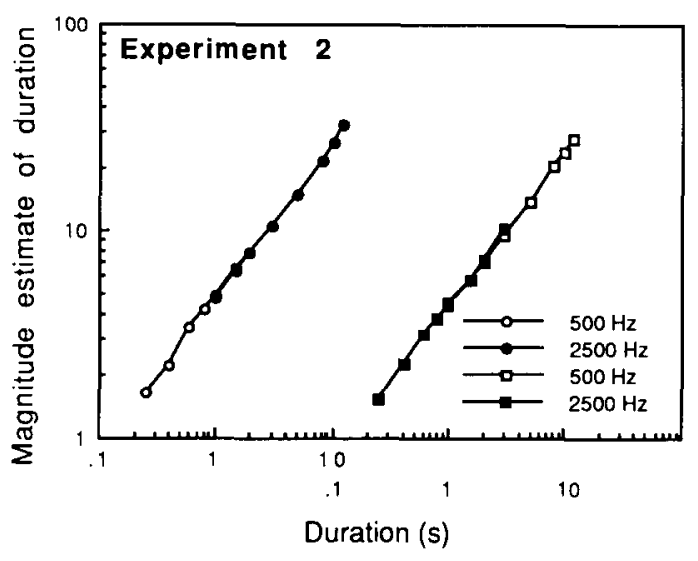

Figure 4. Experiment 2: Geometric average magnitude estimates of the duration of 500-Hz tones (open symbols) and 2500-Hz tones (filled symbols), plotted against physical duration. In Condition A (circles, left), the durations at $500 \mathrm{~Hz}$ were low and those at 2500 Hz were high; in Condition B (squares, right), the durations at $500 \mathrm{~Hz}$ were high and those at $2500 \mathrm{~Hz}$ were low.

this purpose, I used exactly the same sound frequencies, 500 and $2500 \mathrm{~Hz}$, that yielded substantial slippery context effects in judgments of loudness (Marks, 1988; Schneider \& Parker, 1990); see Figure 1. Given the small slippery effects evident in heteromodal judgments of duration (Marks et al., 1986), I suspected that judgments of duration of tones, even ones differing markedly in sound frequency, would reveal little or no such effect.

\section{Method}

Stimuli. Stimuli were 1-sec bursts of tones with approximately sinusoidal waveforms, generated through the sound chip of a Commodore 64 computer, having fundamental frequencies of 500 and $2500 \mathrm{~Hz}$ and presented through Nova-40 headphones at a comfortable level, about $65 \mathrm{~dB}(\mathrm{~A})$. In Condition $\mathrm{A}$, the $500-\mathrm{Hz}$ tones took on low durations $(0.25,0.4,0.6,0.8,1.0,1.5,2$, and $3 \mathrm{sec})$ and the $2500-\mathrm{Hz}$ tones high durations $(1.0,1.5,2,3,5,8,10$, and $12 \mathrm{sec}$ ); in Condition $B$, the durations at each frequency were reversed.

Procedure. The procedure mimicked that of Experiment 1. The subjects gave magnitude estimates of the durations of all of the stimuli in a given condition; the two conditions were tested on different days. Within a session, stimuli were presented in random order, each stimulus being judged a total of 10 times within a session. Sixteen subjects participated, half serving first in Condition $\mathbf{A}$ and then in $\mathbf{B}$, half in $\mathbf{B}$ and then in $\mathbf{A}$.

\section{Results and Discussion}

The average results appear in Figure 4. Much like the judgments of pitch, and quite unlike judgments of loudness, the judgments of duration show no slippery context effect. Perceived duration is essentially unaffected by either sound frequency or contextual condition; instead, perceived duration depends solely on physical duration-here, the magnitude estimates are proportional to about the 0.75 power of stimulus duration.

The absence of slippery context effects for duration suggests again that the presence and size of such effects depend jointly, and thus contingently, on criterial dimen- sion and vehicle. Under otherwise comparable conditions, judgments of duration show no slippery context effect whereas judgments of loudness do.

\section{EXPERIMENT 3 \\ Duration in Two Modalities}

Apparently, judging the durations of stimuli taken from different modalities is not identical to judging the durations of stimuli of different qualities within the same modality. Marks et al. (1986) found a modest but clear-cut differential effect of context on judged duration of visual and auditory stimuli. In that experiment, the visual stimulus took on the same values of duration in both conditions, but the auditory stimulus took on high durations in one condition and low ones in the other. In the former condition, equally long visual and auditory stimuli were judged to be equal; in the latter, they were judged to be unequal.

On the other hand, Marks et al. (1986) found a large slippery context effect when subjects judged the intensity, rather than the duration, of stimuli taken from different modalities-as large an effect as Marks (1988) found when subjects judged loudness of tones differing in sound frequency. Why did Marks et al. (1986) find a slippery context effect with heteromodal stimuli, whereas the present Experiment 2 yielded no effect at all with unimodal stimuli?

The difference may, of course, simply reflect a "looser coupling" in judging heteromodal stimuli, which is perhaps based on a partial but not complete overlap between the mechanisms responsible for judgments of duration presented through different sensory channels (modalities). But another possible explanation is methodological: The difference might reflect procedural variations between the two experiments. First, Marks et al. (1986) kept one of the stimulus sets (visual) constant across the two contextual conditions, while the other set (auditory) varied; in the present Experiment 2, however, both stimulus sets varied across contextual conditions. Second, and perhaps more importantly, in Marks et al.'s (1986) study, the two types of stimulus-tone and light-were presented from trial to trial in strict alternation; only the durations varied randomly over trials. By way of contrast, in the present Experiment 2, quality (sound frequency) as well as duration varied randomly from trial to trial.

Is this methodological difference significant? Marks et al. (1986) suggested that their slippery context effects may have resulted in part from their procedure of trialto-trial alternation between modalities. They based this conjecture on the well-known finding that magnitude judgments of all sorts are susceptible to sequential dependencies: The response to the current stimulus depends not only on the physical characteristics of that stimulus but also on the stimuli and responses in earlier trials. In particular, Ward $(1979,1982,1990)$ has uncovered two kinds of sequential processes. One, assimilation of the current response to prior responses, occurs independently of the quality or modality of the stimuli, whereas the sec- 
ond, contrast to prior stimuli, occurs only within the same sensory modality, and then only with qualitatively similar stimuli. Because the strongest sequential dependencies connect the current trial $i$ to the previous trial $i-1$, the procedure of strictly alternating modalities (or qualities) on successive trials would enhance response assimilation but restrict stimulus contrast. Marks et al. (1986) showed mathematically and by simulation how, in principle, the use of strict alternation by itself could lead to a small slippery context effect through short-term response assimilation. Experiment 3 asked whether judgments of duration would show a slippery context effect when the vehicle was sensory modality rather than pitch, but the procedure was otherwise identical to that of Experiment 2 -that is, when the modality varied randomly from trial to trial.

\section{Method}

The criterial dimension remained duration, but the vehicle was now sensory modality. The auditory stimuli were $500-\mathrm{Hz}$ tones, identical to those in Experiment 2. The visual stimuli were the same as those used by Marks et al. (1986): black rectangles, $1.15 \mathrm{~cm}$ wide $\times 0.75 \mathrm{~cm}$ high, located at the center of a Super Brain color monitor and viewed against a gray background. Otherwise, the stimulus durations and composition of the two stimulus sets, A and B, were identical to those of Experiment 2. Again, 16 subjects judged the durations of 10 replicates of each set of 16 stimuli, 8 auditory durations and 8 visual durations. All subjects served in two sessions, held on different days; half served first in Condition $A$ and then in $B$, half in $B$ and then in $A$.

\section{Results and Discussion}

Figure 5 shows the geometric average results, revealing a small but clear slippery context effect. In Condition A, in which tones took on the low durations and lights the high durations (left side of the figure), tones were estimated to last on the average $21.5 \%$ longer than lights of the same physical duration; but in Condition B, in

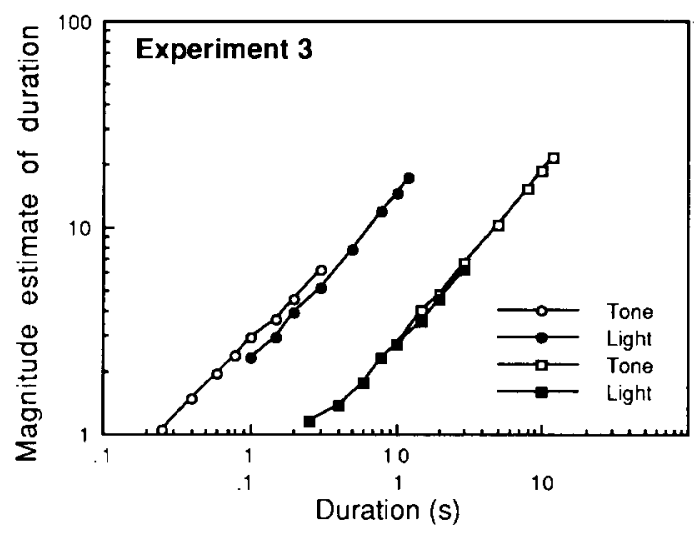

Figure 5. Experiment 3: Geometric average magnitude estimates of the duration of $500-\mathrm{Hz}$ tones (open symbols) and black rectangles (filled symbols), plotted against physical duration. In Condition $A$ (circles, left), the durations of the tone were low and those of the light were high; in Condition B (squares, right), the durations of the tone were high and those of the light were low. which tones took on the high durations and lights the low ones (right side of the figure), tones were judged to last merely $6.5 \%$ longer than lights of the same physical duration.

To evaluate the results statistically, I entered the logarithms of each subject's mean judgments into an analysis of variance (ANOVA), using the three variables of modality (visual vs. auditory), contextual condition (A vs. B), and level $(1,1.5,2$, and $3 \mathrm{sec}$, the four durations common to the two conditions). The ANOVA revealed only two statistically reliable effects: an effect of level, reflecting the increase in judgments with increasing duration $[F(3,45)=233.0, p<.0001]$, and an interaction of modality $\times$ context, reflecting the slippery context effect $[F(1,15)=8.84, p=.01]$. Only one other effect even approached significance: the effect of modality $[F(1,15)=5.99, p=.07]$, reflecting the well-known tendency for subjects to judge auditory durations as longer than corresponding visual durations (e.g., Goldstone \& Goldfarb, 1963).

Evidently, judgments of duration of stimuli from different modalities do display a small but reliable slippery context effect-in contrast with judgments of unimodal (auditory) stimuli, which gave no evidence at all of such an effect. Furthermore, unlike the analogous results of Marks et al. (1986), the present effect with heteromodal stimuli cannot be attributed to differential sequential dependencies. Still, it is interesting that these contextinduced shifts in cross-modally matching durations are substantially smaller than those reported by Marks et al. $-4.7 \%$ versus $22 \%$ of the difference between the mean log durations of the two contextual sets themselves. For example, in the present Condition A, stimulus durations much like those in Marks et al.'s analogous condition were used; yet the effect of context was much greater in Marks et al.'s experiment, probably because of the sequential effects induced in that study through alternation between modalities. Perhaps the shifts found by Marks et al. represent the sum of multiple effects: a small but "pure" slippery context effect; a larger effect, probably based in sequential dependencies; and perhaps also an effect of information about modality provided by the fixed sequence of alternation.

\section{EXPERIMENT 4 Length at Two Colors}

Experiment 4 asked how changes in stimulus context might differentially affect judgments of length. The experiment was motivated by the possibility that judgments of intensity, but not extensity, would show slippery context effects when the vehicle was quality. This possibility was itself suggested by the results of Experiment 2, which showed an absence of slippery context effects on judgments of duration; under comparable circumstances, loudness shows a large slippery context effect. Perhaps, when the vehicle is sensory quality, slippery context effects are largely restricted to judgments of intensity, and 
not extensity - as long as all of the stimuli are presented to a single sensory modality (we may need this proviso, for judgments of heteromqdal stimuli may always show some slippery context effect).

To date, only Potts (1990) has used perceived length as a criterial dimension; the vehicle dimension was spatial orientation (vertical or horizontal) rather than quality of the line segments. Potts found statistically reliable, but relatively small, slippery context effects both when the lines were viewed in normal lighting and when they were viewed in darkness.

The present experiment was designed by way of analogy to Experiment 2 and to the main experiments of Marks (1988). Marks examined how context affected relative loudnesses at two frequencies (pitches), 500 and $2500 \mathrm{~Hz}$; the present Experiment 2 examined how context affected perceived duration at the same two frequencies. Drawing an analogy between pitch in hearing and color in vision, both being qualitative dimensions of perceptual experience, Experiment 4 examined how context might affect the perceived length of visual stimuli presented at two colors.

\section{Method}

Stimuli. The stimuli were horizontal lines, centered at the middle of the $28.5 \times 21.5 \mathrm{~cm}$ screen of a Leedex color monitor. Each line was $1 \mathrm{~mm}$ thick, and could take on either of two colors: green (approximately Munsell $7.5 \mathrm{GY} \mathrm{8/8)}$ and orange (approximately Munsell 2.5 YR 7/12). In Condition A, the green lines were relatively short $(4,8,10,16,20,32,46$, and 70 pixels) and the orange lines relatively long $(20,32,46,70,81,123,165$, and 230 pixels); in Condition B, the sets of lengths were reversed. Each pixel was $0.828 \mathrm{~mm}$ long. Again, note the overlap in the stimulus sets: Four of the lengths were common to all subsets of stimuli.

Procedure. Experiment 4 followed the same magnitude-estimation procedure as that in Experiments 1-3, with subjects instructed to assign numbers to length, entering the responses on a numerical keypad. Each of the 16 stimuli in a given condition was presented a total of 16 times in the session. Each line remained visible on

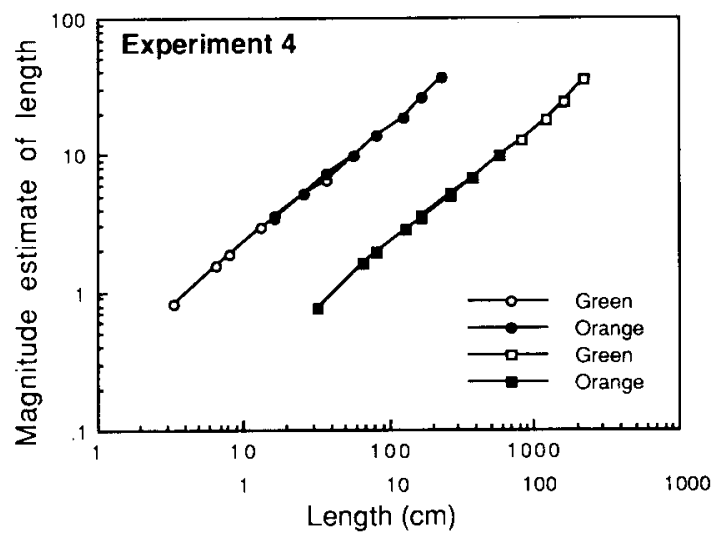

Figure 6. Experiment 4: Geometric average magnitude estimates of the length of green lines (open symbols) and orange lines (filled symbols), plotted against physical length. In Condition A (circles, left), the lengths of the green lines were low and those of the orange lines were high; in Condition B (squares, right), the lengths of the green lines were high and those of the orange lines were low. the screen until the subject entered the response (on the average, for about $5 \mathrm{sec}$ ). Sixteen young men and women served as subjects in two sessions held on separate days; half participated first in Condition $\mathbf{B}$ and then in $\mathbf{A}$, half in $\mathbf{A}$ and then in $\mathbf{B}$.

\section{Results and Discussion}

Geometric mean estimates of length appear in Figure 6, which shows that the dimension of length too evades slippery context effects, at least under the present conditions. The judgments are roughly proportional to physical length (slopes of about 0.9 in these $\log$ - $\log$ coordinates) and are wholly independent of both the color and the contextual set of levels. Thus the results are consistent with the hypothesis that slippery context effects are absent in judgments of extensive dimensions (length, duration), when the vehicle for contextual variation is qualitative (color, pitch).

\section{EXPERIMENT 5}

\section{Absolute Magnitude Estimation of Loudness}

The first four experiments showed slippery context effects to be largely absent from judgments of pitch when the vehicle dimension was intensity, and from judgments of duration and length when the vehicle was quality; earlier studies (Marks, 1988; Schneider \& Parker, 1990) showed large slippery context effects in judgments of loudness when the vehicle was quality. Hence slippery context effects are not universal characteristics of quantitative judgments of multidimensionally varying stimuli-perhaps because these effects arise not from changes in response processes alone (which tend to be general), but from changes in perception.

Schneider and Parker (1990) obtained analogous effects with a procedure in which, on each trial, subjects merely decided which of two pairs of tones differed more in loudness, from which they suggested that context affected not only overt judgments, but the underlying loudness percepts themselves. Later, Marks (1991b) reported that direct loudness matches between 500 - and $2500-\mathrm{Hz}$ tones changed when matching trials were embedded within scaling sessions with different contextual stimulus sets. Algom and Marks (1990) came to a related conclusion, which they based on their finding that changes in context modified the rules governing such fundamental processes as binaural summation and temporal summation.

If slippery context effects represent changes in perception, not just changes in overt responses, then slippery effects should persist in magnitude estimation even if judgmental biases can be minimized or eliminated. In this regard, Zwislocki (1983; Zwislocki \& Goodman, 1980) has advocated a method he calls absolute magnitude estimation (hereafter, AME). According to Zwislocki, each person develops a unique coupling between the internal magnitudes of numbers and the magnitudes of sensations. Given appropriate instructions, subjects can reveal these "absolute" couplings and thereby avoid many of the contextual biases that otherwise plague numerical judgments. To this end, Zwislocki recommends instructions that avoid reference to ratios or proportions but instead require sub- 
jects to try to match numbers to sensations. Although Ward (1987) found residual context effects and sequential dependencies evident even in AME, and although Foley, Cross, and O'Reilly (1990) reported clear effects of context with AME instructions, AME may serve to reduce-though surely not to eliminate-response-based effects of context (Gescheider \& Hughson, 1991; Zwislocki \& Goodman, 1980).

Nevertheless, as already indicated, slippery context effects may derive not from response-based processes but from changes in underlying percepts. In previous studies with AME, test conditions contained only unidimensionally varying stimuli, such as, for example, different sets of SPLs at a single sound frequency. By way of contrast, slippery context effects refer to changes obtained in test conditions with multidimensionally varying stimuli, such as, for example, different sets of SPLs at different sound frequencies. The use of multidimensionally varying stimulus contexts may be crucial to the generation of perceptual changes-if slippery context effects do represent changes in perception.

Experiment 5 applied the method of AME to judgments of loudness of 500 - and $2500-\mathrm{Hz}$ tones, repeating as closely as possible Marks's (1988) Experiment 3, except for a change in instructions to the subjects. If changing instructions does eliminate slippery context effects, it seems reasonable to infer that they arise from "biases" in judgment (i.e., from different response functions applied to the different sound frequencies). But if they resist changing instructions, the results, though clearly less decisive, would be consistent with the hypothesis that slippery context effects are substantially perceptual in nature (such results would also be consistent, however, with the hypothesis that slippery context effects are judgmental, but that AME does not eliminate judgmental "biases").

\section{Method}

Stimuli. The stimuli were pure tones of 500 and $2500 \mathrm{~Hz}$, like those in Experiment 1. Each tone lasted $1 \mathrm{sec}$, with rise and decay of $10 \mathrm{msec}$. In Condition $\mathrm{A}$, the $500-\mathrm{Hz}$ tones were low (eight SPLs from 35 to $70 \mathrm{~dB} \mathrm{SPL}$ in steps of $5 \mathrm{~dB}$ ) and the $2500-\mathrm{Hz}$ tones were high (eight SPLs from 50 to $85 \mathrm{~dB}$ in steps of $5 \mathrm{~dB}$ ); in Condition $\mathrm{B}$, the $500-\mathrm{Hz}$ tones were high $(55-90 \mathrm{~dB})$ and the $2500-\mathrm{Hz}$ tones low $(30-65 \mathrm{~dB})$.

Procedure. The method consisted of absolute magnitude estimation. The subjects read the following instructions:

\footnotetext{
This is an experiment that concerns the perception and judgment of loudness. On each trial you will hear a tone, presented briefly (for about $1 \mathrm{sec}$ ), after which you will try to judge its loudness numerically. To do this, you should try to give to the loudness of each tone the number that seems best to "match." If the sound is soft, give a small number. If the sound is loud, give a big number. Use your own sense of what is small and what is big. And don't worry about being consistent with numbers you may have given on previous trials. Just treat each trial as independent and on each trial try to give the best "absolute match" of number to loudness.
}

As in Marks's (1988) Experiment 3, each of the 16 stimuli in a given condition, $A$ or $B$, was presented a total of 16 times, with every stimulus following every stimulus including itself exactly once. Again 16 men and women participated, half serving first in Condition $B$ and then in $A$, and half in $A$ and then in $B$.

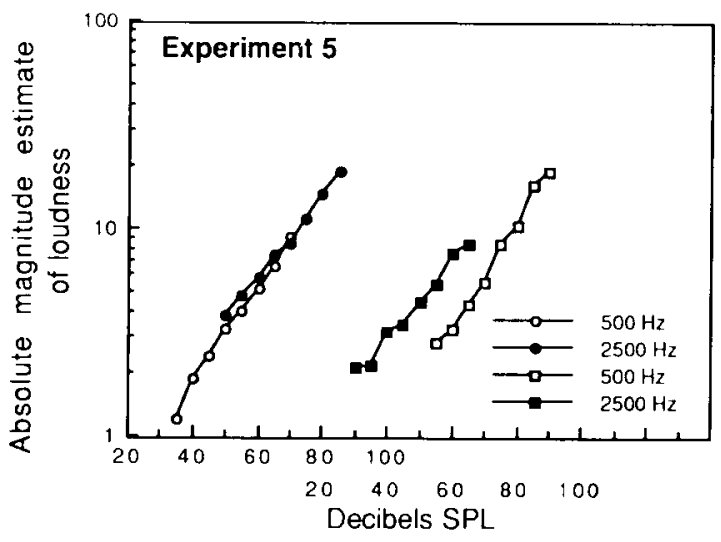

Figure 7. Experiment 5: Geometric average absolute magnitude estimates of the loudness of 500-Hz tones (open symbols) and 2500Hz tones (filled symbols), plotted agginst sound-pressure level (SPL). In Condtion A (circles, left), the SPLs at $500 \mathrm{~Hz}$ were low and those at $2500 \mathrm{~Hz}$ were high; in Condition B (squares, right), the SPLs at $500 \mathrm{~Hz}$ were high and those at $500 \mathrm{~Hz}$ were low.

\section{Results and Discussion}

The pooled data appear in Figure 7. Compare this figure with Figure 1, which shows the analogous results obtained earlier with magnitude-estimation instructions that did refer to proportions and did not emphasize absolute matches between sensations and numbers (Marks, 1988, Experiment 3). Like the earlier study, the present experiment produced a substantial slippery context effect. In Condition A of both experiments (low SPLs at $500 \mathrm{~Hz}$ and high SPLs at $2500 \mathrm{~Hz}$; left side of the figures), the two loudness functions largely overlap; that is, in Condition A, subjects judged $2500-\mathrm{Hz}$ tones to be as loud as $500-\mathrm{Hz}$ tones of the same SPL. But in Condition B of both experiments (high SPLs at $500 \mathrm{~Hz}$, low at $2500 \mathrm{~Hz}$; right side of the figures), the loudness function at $2500 \mathrm{~Hz}$ sits well above the function at $500 \mathrm{~Hz}$; that is, in Condition B, subjects judged $2500-\mathrm{Hz}$ tones to be considerably louder than $500-\mathrm{Hz}$ tones of the same SPL.

Although Marks's (1988) Experiment 3 and the present Experiment 5 yielded similar pictures, there is a quantitative difference: The slippery context effect was larger in the earlier study, in which the change in stimulus context resulted in an average shift of $17 \mathrm{~dB}$ in the SPLs at 500 and $2500 \mathrm{~Hz}$ judged to be equally loud; in the present experiment, the same change in context resulted in an average shift of only about $12 \mathrm{~dB}$.

Because the present Experiment 5 and Marks's (1988) Experiment 3 were the same except for the instructions, it is reasonable to enter the results (log judgments) from the two experiments into an overall ANOVA, using the between-subject variable of experiment, and the withinsubject variables of SPL, frequency, and contextual condition. The ANOVA showed all main effects to be reliable: intensity $[F(3,90)=175.1, p<.0001$; loudness judgments increase with SPL]; frequency $[F(1,30)=$ $7.52, p=.01$; greater judgments at 2500 than at $500 \mathrm{~Hz}$; 
context $[F(1,30)=4.38, p=.045$; greater judgments in Condition B]; and experiment $[F(1,30)=9.49, p=$ .0044; greater judgments-in AME]. The ANOVA showed three reliable interactions: context and frequency $[F(1,30)$ $=118.5, p<.0001$; the overall slippery context effect]; frequency and intensity $[F(3,90)=7.94, p=.0001$; greater slope at $2500 \mathrm{~Hz}$; and experiment, frequency, and intensity $[F(3,90)=3.025, p=.034$; smaller slope difference with AME]. Most importantly, the three-way interaction of contextual condition, frequency, and experiment was not reliable $[F(1,30)=1.29, p=.26]$. Thus even though the slippery context effect is numerically smaller with AME, it is not possible to conclude from these results that the change in instructions reduced the size of the effect-even though the outcome is suggestive. In any case, even with AME, the slippery context effect is substantial.

\section{EXPERIMENT 6 Effect of Cuing the Vehicle}

Let me pick up here a promissory note: In the introduction to Experiment 3, I pointed out that in some studies on effects of multiple contexts, stimuli alternated from trial to trial between two modalities (e.g., Marks et al., 1986) or two qualities (Marks, 1988, Experiment 1). In comparison with a procedure in which stimuli are randomized with respect to quality as well as intensity, the procedure of alternation introduces at least two possible additional effects: differential sequential dependencies (if Quality 1 contains low stimulus levels and Quality 2 high ones, trials on Quality 1 on the average follow high-level stimuli and responses, whereas trials on Quality 2 on the average follow low-level stimuli and responses); and reduced uncertainty (subjects know which quality will appear on every trial).

Marks et al. (1986) noted that the sequential dependencies introduced by stimulus alternation could by themselves produce a small slippery context effect, since the predominant component to sequential dependencies is assimilation of current response to prior response, and Marks (1988, Experiment 2) confirmed the presence of such a sequence-induced effect by alternating between intensity sets of a $500-\mathrm{Hz}$ tone. On the other hand, the introduction of trial-by-trial information about stimulus quality might act to reduce the slippery context effectif, for example, the slippery effect depends at least partly on directed attention. It would be helpful to assess independently the possible roles of sequential dependencies and directed attention.

Do slippery context effects arise automatically and "preattentively"? Or do they depend in some way on attention? Experiment 6 pursued this line of inquiry by examining the role of trial-by-trial information about sound frequency within the double shifting-context paradigm: Again, subjects gave magnitude estimates of the loudness of $500-$ and $2500-\mathrm{Hz}$ tones, in two contextual conditions. Prior to each stimulus presentation, the subject was cued

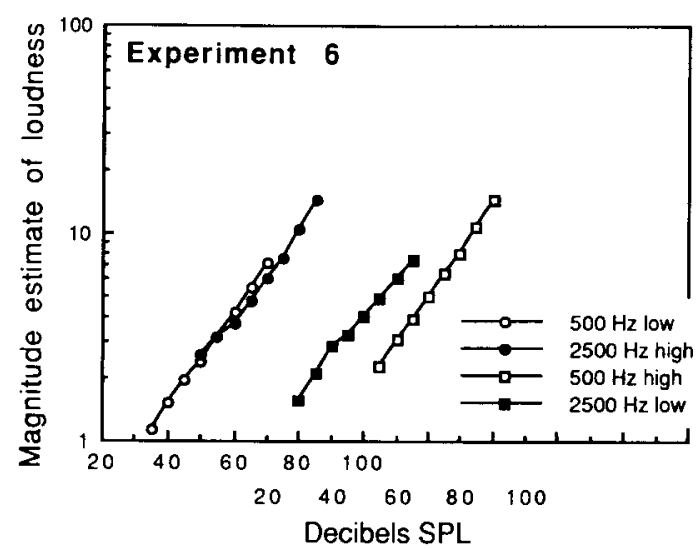

Figure 8. Experiment 6: Geometric average magnitude estimates of the loudness of 500- $\mathrm{Hz}$ tones (open symbols) and $2500-\mathrm{Hz}$ tones (filled symbols), plotted against sound-pressure level (SPL), when the pitch of each tone was cued beforehand visually. In Condition A (circles, left), the SPLs at $500 \mathrm{~Hz}$ were low and those at $2500 \mathrm{~Hz}$ were high; in Condition B (squares, right), the SPLs at $500 \mathrm{~Hz}$ were high and those at $2500 \mathrm{~Hz}$ were low.

visually as to whether the pitch would be low or high, thereby directing attention without using regular stimulus sequences.

\section{Method}

The stimuli, contexts, and procedures were identical to those in Experiment 5, including randomization of SPLs and frequencies, with three exceptions. First, the method was "traditional" magnitude estimation (as in Experiments 1-4 and in Marks, 1988). Second, 1 sec before each stimulus was presented, there appeared at the center of the computer monitor the word "low" or "high," indicating whether the signal would be 500 or $2500 \mathrm{~Hz}$. The word remained on the screen for $2 \mathrm{sec}$-that is, until the tone terminated. Third, an extra 32 trials were inserted at the start of each session. Although not identified as such, these trials served as "practice" for the subjects to learn the auditory referents for the words "low" and "high." Responses to these initial 32 stimuli were not recorded or included in the results.

\section{Results and Discussion}

Figure 8 shows the presence of a large slippery context effect. In fact, the effect is just as large (mean shift in matching SPLs of $17.4 \mathrm{~dB}$ ) as it was in Marks's (1988) comparable Experiment 3; an ANOVA directly comparing the two sets of results showed no interaction of experiment with context and frequency $[F(1,30)<1]$. Clearly there is no evidence that directing attention to the signal frequency on each trial-cuing the subjects to its pitch — had any effect at all on the slippery context effect.

\section{EXPERIMENT 7 Role of Sequential Processes}

The results of Experiment 6 imply that directing attention to sound frequency on each trial has little or no effect on the way in which judgments of loudness at different sound frequencies depend on context. If so, it is likely 
that any difference in results obtained when qualities (or modalities) alternate from trial to trial and when they appear randomly from trial to trial can be ascribed to the differential presence of sequential dependencies (mainly assimilation) in the former variant of the paradigm. Thus, during alternation between a low-frequency tone at low SPLs and a high-frequency tone at high SPLs, the tendency to assimilate the previous response to the current one will raise the responses to the low-frequency tones (because the previous SPL on the average was high) and lower the responses to the high-frequency tones (because the previous SPL on the average was low).

Note, however, that the only direct empirical evidence demonstrating something like a slippery context effect from sequential dependencies comes from Marks's (1988) Experiment 2, which used a single sound frequency, $500 \mathrm{~Hz}$, but which alternated from trial to trial between SPLs selected from low and high sets. This paradigm produced a small differential context effect, where none would be possible if all stimuli were randomized.

Given this result, it is curious that Marks (1988) found the magnitude of the slippery context effect in judgments of loudness of 500 - and $2500-\mathrm{Hz}$ tones to be virtually identical when frequencies alternated from trial to trial and when frequency was randomized. Clearly, some other process wholly apart from sequential assimilation is responsible for the substantial slippery effect that appears when subjects judge loudnesses of tones differing greatly in quality. Perhaps the process that generates substantial slippery context effects, whatever it may be, can overwhelm any possible contribution by sequential assimilation.

In Experiment 7, subjects again judged the loudness of two contextual sets of 500 - and $2500-\mathrm{Hz}$ tones-a condition that reliably produces robust slippery context effects. Under such conditions, neither sequential dependencies nor information about frequency on each trial seems likely to play a substantial role. But will they play any role at all? To help answer this question, Experiment 7 used stimulus sequences modeled after those of Ward (1990); in order to compare sequential effects between successive tones that could be the same or different in frequency, Ward used stimulus sequences in which the same frequency was presented on two successive trials before alternating. Thus, if we designate the low frequency as $\mathrm{L}$ and the high frequency as $\mathrm{H}$, and the first and second presentations in each pair of trials as 1 and 2 , the sequences would be: L1-L2-H1-H2-L1-L2-H1-H2-...

The present experiment applied such stimulus sequences to loudness judgments of 500 - and $2500-\mathrm{Hz}$ tones. Let us consider the consequences for (1) trial-to-trial information about sound frequency, and (2) sequential effects. First, by being regular, these sequences could inform the subject about pitch on each trial. Furthermore, because each frequency was repeated before alternating, the pitch on each second presentation ( $\mathrm{L} 2$ or $\mathrm{H} 2$ ) was cued directly by the pitch on the previous trial ( $\mathrm{Ll}$ or $\mathrm{H} 1$ ). One might argue that Experiment 6 "failed" because people simply are not capable of using pitch information cued lexically; perhaps, though, information helps if the cue is a tone of the same sound frequency-as when cuing affects the detectability of expected as opposed to unexpected signal frequencies (see, e.g., Greenberg \& Larkin, 1968).

Second, note that sequential dependencies will be markedly different on the first and second presentations of a given sound frequency. Consider the case in which lowfrequency tones, L, take on low SPLs, and high-frequency tones, $\mathrm{H}$, high SPLs. On $\mathrm{L} 1$, a tendency to assimilate the response to the previous trial will augment responses (since $\mathrm{H} 2$ comprises high SPLs), and, on $\mathrm{H} 1$, the tendency will diminish responses (since L2 comprises low SPLs); consequently, assimilation should increase the size of the slippery context effect on $\mathrm{L} 1$ and $\mathrm{H} 1$ trials. But on L2 (and H2) trials, a tendency toward assimilation will have the opposite effect, since L1 comprises low SPLs (and $\mathrm{H} 1$ high ones); consequently, sequential assimilation should decrease the size of the slippery effect on $\mathrm{L} 2$ and $\mathrm{H} 2$ trials. (Here I am ignoring propagation of effects across more than one trial, e.g., trial $i-2$ affects trial $i-1$ and hence, indirectly, trial $i$.)

In sum, if information about signal frequency can serve to focus attention, reduce uncertainty, and thereby reduce slippery context effects, the slippery effect should be smaller in the present paradigm than it is when frequency is randomized, and in particular, it might be smaller on the second of each successive pair of stimulus presentations at a given frequency. But if sequential processes contribute substantially, slippery context effects should be enhanced on the first presentation and reduced on the second presentation of each frequency. Clearly, both the informational and the sequential effects go partly in the same direction. On the other hand, if neither cues about pitch nor trial-to-trial sequential effects matter much, the slippery context effect should be essentially the same on the first and second presentations of each frequency in the sequences.

\section{Method}

Stimuli, contexts, and procedures were identical to those of Experiment 5 (AME), with two exceptions. The instructions were those for "traditional" magnitude estimation (as in Experiments 1-4 and 6 ), and the stimulus sequences were orderly, in that the same frequency, 500 or $2500 \mathrm{~Hz}$, was used on two consecutive trials before changing. In each contextual condition, half of the subjects received the low-frequency tone on the first trial, and half the highfrequency tone.

\section{Results and Discussion}

Figure 9 shows the results obtained in each contextual condition, with separate loudness functions plotted for Trials L1 and L2 (first and second presentations of lowfrequency tones in each pair) and for Trials $\mathrm{H} 1$ and $\mathrm{H} 2$ (first and second presentations of high-frequency tones). Although only slightly, the results for first and second presentations do differ, in that the judgments given to the low set of SPLs are a bit higher on the second presentation at each frequency than they are on the first, and the 


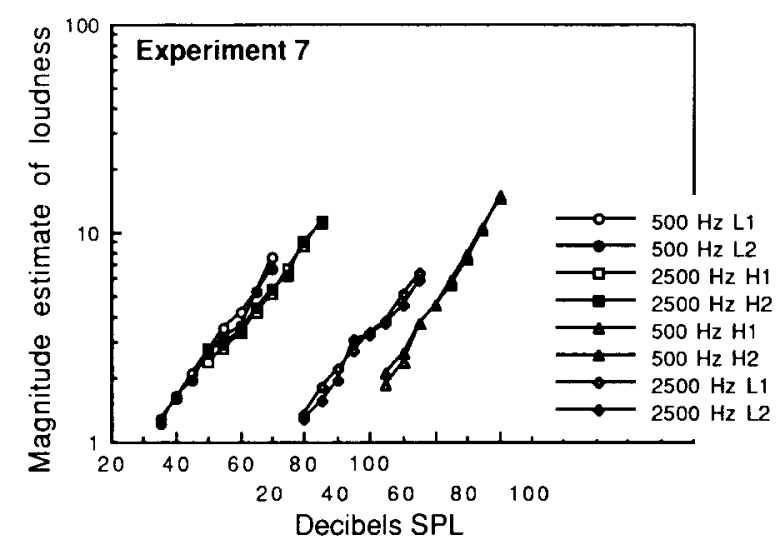

Figure 9. Experiment 7: Geometric average magnitude estimates of the loudness of 500-Hz tones (circles, triangles) and 2500-Hz tones (squares, diamonds), plotted against sound-pressure level (SPL). In the stimulus sequences, each frequency was repeated on two trials before changing; open and flled symbols give results for Presentations 1 and 2, respectively. In Condition $A$ (circles and squares, left), the SPLs at $500 \mathrm{~Hz}$ were low (L) and those at $2500 \mathrm{~Hz}$ were high (H); in Condition B (triangles and diamonds, right), the SPLs at $500 \mathrm{~Hz}$ were high and those at $2500 \mathrm{~Hz}$ were low.

judgments given to the high set of SPLs are a bit lower on the second presentation than they are on the first. This modest effect appears in both contexts, and it acts to reduce the overall size of the slippery context effect from $20.3 \mathrm{~dB}$ (first presentations) to $18.8 \mathrm{~dB}$ (second presentations). Small as these differences are, given the sensitive within-subject design, an ANOVA showed them to be highly reliable $[F(1,30)=26.3, p=.0001]$.

It is interesting that the slippery context effect on the first presentations of each sound frequency is the largest that I have yet found in judgments of loudness given these sound frequencies and contextual sets of SPLs; even on the second presentations, the context effect was numerically slightly greater than that obtained in Experiment 6 (when pitch was cued visually) and under comparable conditions when pitch was not cued (Marks, 1988, Experiment 3). Most likely, the stimulus sequences used in Experiment 7 did modulate the underlying slippery context effects by dint of the differential serial dependencies that the sequences produced. It seems improbable that trialto-trial information about pitch, even on the second presentations, plays a substantial role.

\section{GENERAL DISCUSSION}

\section{Slippery Context Effects and \\ Perceptual Encoding}

Why should the presence and magnitude of slippery context effects depend jointly, in the way they do, on the criterial dimension judged and the vehicle through which the stimulus sets change contextually? Simple distinctions like that between prothetic and metathetic continua fail because loudness and duration are both prothetic, yet loudness shows slippery effects and duration does not, given the same vehicle of pitch. Nor does the distinction between intensive and extensive continua per se help, for length judgments show reliable slippery context effects when the vehicle is spatial orientation, but not when the vehicle is color.

When slippery context effects do appear, they imply that subjects are using a relativistic code in addition to an absolute code for perception and/or judgment. One possibility is that a relativistic code comes into play when subjects must compare information transmitted along different processing subchannels. That is, slippery context effects may depend jointly on the criterial dimension and vehicle because, or to the extent that, the criterial dimension is encoded in a processing channel that is not unitary, but "distributed" across the vehicle. Thus, loudness judgments at 500 and $2500 \mathrm{~Hz}$ may show a slippery context effect because (1) listening to tones of very different sound frequencies leads to the activation of essentially distinct subsets of "neural units" (distinct subchannels), and (2) the comparison of levels of activity (loudness) across different subchannels necessarily invokes contextual or relativistic information. That is, comparison of loudnesses across critical bands (subchannels) may require the use of a context-coding mechanism, like that postulated by Durlach and Braida (1969; see especially Braida et al., 1984), as well as a sensory-coding mechanism.

By contrast, to the extent that pitch (at least, spectral pitch) is encoded by a unitary system (e.g., "neural units" firing at characteristic frequency, independently of sound intensity), changes in loudness should not invoke this kind of contextual processing of pitch; judgments of any given pitch at different loudnesses will rely on comparisons of levels of activity within a single channel, and hence no slippery context effect will appear.

When subjects judged the duration of tones at different sound frequencies (Experiment 2), they faced a situation analogous to that faced by Marks's (1988, Experiment 3) subjects when they judged loudness at different frequencies (and by the subjects of the present Experiment 5). But duration, at least within the auditory system, is presumably not encoded within individual frequency subchannels or critical bands, but in a higher level, and presumably unitary, system. In fact, many theories of time perception and time discrimination propose a common central mechanism (see Allan, 1979). The presence of a slippery context effect when subjects judge the durations of stimuli in different modalities implies that the auditory and visual systems provide only partially overlapping or common, central systems for the judgment of time.

Finally, this hypothesis is at least reasonably compatible with findings on judgments of length; perceived length remains free from effects of contextual changes of length with color (Experiment 4), though not wholly free from contextual changes of length with spatial orientation (Potts, 1990) which is consistent with the view that information about length is processed by a visual subsystem that "ignores" color (that is, there are not individual chromatic subchannels for length), but that does not "ignore" orientation 
(that is, there are overlapping but not identical subchannels for horizontally and for vertically oriented line segments).

\section{Slippery Context Effects Are "Automatic"}

Finally, the evidence at hand suggests that slippery context effects occur " automatically," at a preattentive level of processing. It seems hardly to matter whether a subject knows on a given trial what value the stimulus will have on the vehicle dimension (e.g., what the pitch will be when the criterial dimension is loudness). This automaticity is compatible with, though it does not entail, the distinct likelihood that slippery context effects represent changes in perceptual representations, not just in responding or the mapping of quantitative responses onto underlying percepts (Marks, 1991b; Schneider \& Parker, 1990)that changes in loudness matches across sound frequency, for example, are not the result of some variation in response "bias," but instead reflect changes in loudness itself. (Indeed, the specificity and asymmetry of slippery context effects make them reminiscent of other kinds of contingent perceptual interactions, such as the orientationspecific aftereffects in color of spatial gratings reported by McCollough, 1965-though the two effects probably rely on rather different mechanisms.) Under such an interpretation, the perception of even so seemingly simple and "basic" a property as loudness depends on the stimulus set, and perhaps on "expectations." Consequently, the judgment of loudness is most appropriately viewed not as a window on relatively low-level sensory processes, but as a process that fundamentally entails the properties of a cognitive act.

\section{REFERENCES}

Algom, D., Marks, L. E. (1990). Range and regression, loudness scales and loudness processing: Toward a context-bound psychophysics. Joumal of Experimental Psychology: Human Perception \& Performance, 16, 706-721.

Allan, L. G. (1979). The perception of time. Perception \& Psychophysics, 26, 340-354.

Braida, L. D., Lim, J. S., Berliner, J. E., Durlach, N. I., Rabinowitz, W. M., PuRKs, S. R. (1984). Intensity perception: XIII. Perceptual anchor model of context-coding. Journal of the Acoustical Society of America, 76, 722-731.

Cross, D. V. (1973). Sequential dependencies and regression in psychophysical judgments. Perception \& Psychophysics, 14, 547-552.

Durlach, N. I., \& Bradda, L. D. (1969). Intensity perception: I. Preliminary theory of intensity resolution. Journal of the Acoustical Society of America, 46, 372-383.

Foley, H. J., Cross, D. V., \& O'Reilly, J. A. (1990). Pervasiveness and magnitude of context effects: Evidence for the relativity of absolute magnitude estimation. Perception \& Psychophysics, 48, 551-558.

GARNER, W. R. (1974). The processing of information and structure. Potomac, MD: Erlbaum.

Gescheider, G. A., \& Hughson, B. A. (1991). Stimulus context and absolute magnitude estimation: A study of individual differences. Perception \& Psychophysics, 50, 45-57.

Goldstone, S., \& GoldFARB, J. (1963). Judgments of filled and unfilled durations: Intersensory effects. Perceptual \& Motor Skills, 17, 763-774.

Grau, J. W., Kemler Nelson, D. G. (1988). The distinction be- tween integral and separable dimensions: Evidence for the integrality of pitch and loudness. Journal of Experimental Psychology: General, 117, 347-370.

Greenberg, G. Z., LARKIN, W. D. (1968). Frequency-response characteristics of auditory observers detecting signals of a single frequency in noise: The probe-tone method. Joumal of the Acoustical Society of America, 44, 1513-1523.

HeLson, H. (1964). Adaptation-level theory: An experimental and systematic approach to behavior. New York: Harper \& Row.

JesteAdT, W., LuCE, R. D., \& GREen, D. M. (1977). Sequential effects in judgments of loudness. Joumal of Experimental Psychology: Human Perception \& Performance, 3, 92-104.

LoCKHeAD, G. R., Hinson, J. (1986). Range and sequence effects in judgment. Perception \& Psychophysics, 40, 53-61.

MArKs, L. E. (1988). Magnitude estimation and sensory matching. Perception \& Psychophysics, 43, 511-525.

MARKs, L. E. (1991a). Reliability of magnitude matching. Perception \& Psychophysics, 49, 31-37.

MARKs, L. E. (1991b, March). Slippery context effects in psychophysical judgement. Paper presented to the Experimental Psychology Society, Cambridge, U.K.

Marks, L. E., Stevens, J. C., Bartoshuk, L. M., Gent, J. F., RifKIN, B., \& STONE, V. K. (1988). Magnitude-matching: The measurement of taste and smell. Chemical Senses, 13, 63-87.

Marks, L. E., Szczesiul, R., Ohlott, P. (1986). On the crossmodal perception of intensity. Joumal of Experimental Psychology: Human Perception \& Performance, 12, 517-534.

MARKs, L. E., WARNER, E. (1991). Slippery context effects and critical bands. Joumal of Experimental Psychology: Human Perception \& Performance, 17, 986-996.

McCollough, C. (1965). Color adaptation of edge-detectors in the human visual system. Science, 149, 1115-1116.

Melara, R. D., Marks, L. E. (1990). Perceptual primacy of dimensions: Support for a model of dimensional interaction. Joumal of Experimental Psychology: Human Perception \& Performance, 16, 398-414. PARDucCI, A. (1965). Category judgment: A range-frequency model. Psychological Review, 72, 407-418.

PotTs, B. C. (1990). The horizontal-vertical illusion: A confluence of configural, contextual, and framing factors. Unpublished doctoral dissertation, Yale University.

SCHNEIDER, B., \& PARKer, S. (1990). Does stimulus context affect loudness or only loudness judgment? Perception \& Psychophysics, 48, 409-418.

Stevens, S. S. (1935). The relation of pitch to intensity. Journal of the Acoustical Society of America, 6, 150-154.

WARD, L. M. (1979). Stimulus information and sequential dependencies in magnitude estimation and cross-modality matching. Joumal of Experimental Psychology: Human Perception \& Performance, 5, 444-459.

WARD, L. M. (1982). Mixed-modality psychophysical scaling: Sequential dependencies and other properties. Perception \& Psychophysics, 31, 53-62.

WARD, L. M. (1987). Remembrance of sounds past: Memory and psychophysical scaling. Joumal of Experimental Psychology: Human Perception \& Pefformance, 13, 216-227.

WARD, L. M. (1990). Critical bands and mixed-frequency scaling: Sequential dependencies, equal-loudness contours, and power function exponents. Perception \& Psychophysics, 47, 551-562.

ZwISLOCKI, J. J. (1983). Group and individual relations between sensation magnitudes and their numerical estimates. Perception \& Psychophysics, 33, 460-468.

ZwislockI, J. J., Goodman, D. A. (1980). Absolute scaling of sensory magnitudes: A validation. Perception \& Psychophysics, 28, 28-38.

\section{NOTE}

1. With regard to the possible role of musical training, the subjects in this experiment ranged widely in extent of their prior musical ex- 
perience. Several had virtually no musical training (no formal lessons, no experience outside of grade school music), and others had musical avocations (they were teachers of music). To assess any possible relation of musical training to performance, I divided the subjects into three groups: low musical training (less than 3 years of lessons or equivalent experience with musical instrument or voice); medium training ( 3 to 8 years); and high training (more than 8 years). In both Experiments $1 \mathrm{~A}$ and $1 \mathrm{~B}$, subjects with greater musical training did show a smaller tendency to assimilate loudness to pitch. That is, musically trained subjects tended to judge pitch solely as a function of frequency, not as a joint function of frequency and intensity. Unfortunately, the variability within each subgroup was substantial, so in neither experiment were the differences among subgroups reliable $[F(2,13)<1$ in both].

(Manuscript received August 27, 1990; revision accepted for publication September 12, 1991.) 